\title{
Development of a packaging cell line for propagation of replication-deficient adenovirus vector
}

\author{
Jong-Sik Kim ${ }^{1,3}$, Seung-Hoon Lee ${ }^{2}$, \\ Yong-Suk $\mathrm{Cho}^{2}$, Kyoungsook Park ${ }^{2}$, \\ Young $\mathrm{Ho} \mathrm{Kim}^{3}$ and Je-Ho Lee, \\ ${ }^{1}$ Clinical Research Center, Samsung Biomedical Research
Institute, Seoul 135-710, Korea
${ }^{2}$ Molecular Therapy Research Center, Sungkyunkwan University,
Samsung Medical Center Annex 8F, llwon-dong 50, Kangnam-ku,
Seoul 135-710, Korea
${ }^{3}$ Department of Microbiology, College of Natural Sciences,
Kyungpook National University, Taegu 702-701, Korea
${ }^{4}$ Corresponding author: Tel, +82-2-3410-3510;
Fax, +82-2-3410-0044; E-mail: jeholee@ @amsung.co.kr
}

Accepted 15 September, 2001

Abbreviations: RCV, replication-competent virus; HeLa-E1, HeLa cell that expresses adenovirus E1 gene; Ad, adenovirus; m.u., map unit; m.o.i, multiplicity of infection

\begin{abstract}
A human embryonic kidney cell line 293 is widely used for adenovirus production and propagation. With this cell line, however, replication-competent virus (RCV) is frequently generated, especially during large-scale production and successive propagation because 293 cells contain not only E1 gene but also non-E1 adenovirus gene. Homologous recombination between non-E1 region of 293 genomic DNA and its homologous region in the recombinant adenoviral vector generate RCV. To overcome this problem, we developed a new packaging cell line, Hela-E1, which contains minimum $\mathrm{E} 1$ region and from which non-E1 adenoviral region that is homologous with recombinant adenovirus vector was excluded. No RCV was detected during adenovirus propagation in Hela-E1 compared to in 293. In addition, adenovirus-p53 produced in HeLa-E1 was able to overexpress p53 protein when introduced into an ovarian cancer cell line, SKOV3. These results may have a significant impact on the development of packaging cell lines for replication-deficient adenovirus production.
\end{abstract}

Keywords: adenovirus; replication competent virus; packaging cell line; HeLa-E1 cell

\section{Introduction}

A recombinant adenovirus vector has been extensively utilized as a delivery vehicle for gene transfer, vaccination, and gene therapy (Zhang, 1999). The widely used recombinant adenovirus vector was derived from subgroup $\mathrm{C}$ and is highly infectious and capable of delivering therapeutic genes to many different cell types. Among subgroup $C$, the serotypes 2 and 5 have been used for vector development because they are not associated with any severe pathology in humans (Horwitz, 1990). In some cases, however, the generation of RCV (replication-competent virus) has been a major obstacle in production and successive propagation of adenovirus vectors because it causes cell toxicity by itself and leads to immunological response (Smith et al., 1993; Yang et al., 1994).

The general method to obtain recombinant adenoviruses involves homologous recombination in packaging cells capable of complementing defective adenoviruses (Berkner and Sharp, 1983). Homologous recombination results in generation of a replication-defective adenovirus that can not replicate in host cells unless the E1 gene critical for adenovirus replication is supplied. However, RCV production was often detected during a large-scale adenovirus propagation or successive production in universal packaging cell line, 293 (Lochmuller et al., 1994). This is due to a fact that 293 cells contain and express the left $11 \%$ of the Adenovirus type5 (Ad5) genome and this region contains a part of adenovirus $E 1$ region (1.6-9.7 m.u.) as well as non-E1 region (0-1.6 m.u., 9.7-11 m.u.). RCV is presumably generated by the homologous recombination between non-E1 region of 293 genomic DNA and homologous region of a recombinant adenovirus vector (Figure 1).

In the present study, we reported a strategy for making a new packaging cell line, HeLa-E1, which does not contain non-E1 region but a minimum E1 gene (1.69.7 m.u.) of adenovirus type 5 (Ad5). We also demonstrated that no RCV was generated during adenovirus production in HeLa-E1 as compared to in 293. Moreover, Adenovirus-p53 (Ad-p53) produced in HeLa-E1 induced an overexpression of p53 protein when transfected into an ovarian cancer cell line, SKOV3. These results indicate that HeLa-E1 can act as a packaging cell line that excludes RCV generation and the strategy used here can be useful for making other new packaging cell lines for recombinant adenovirus production. 


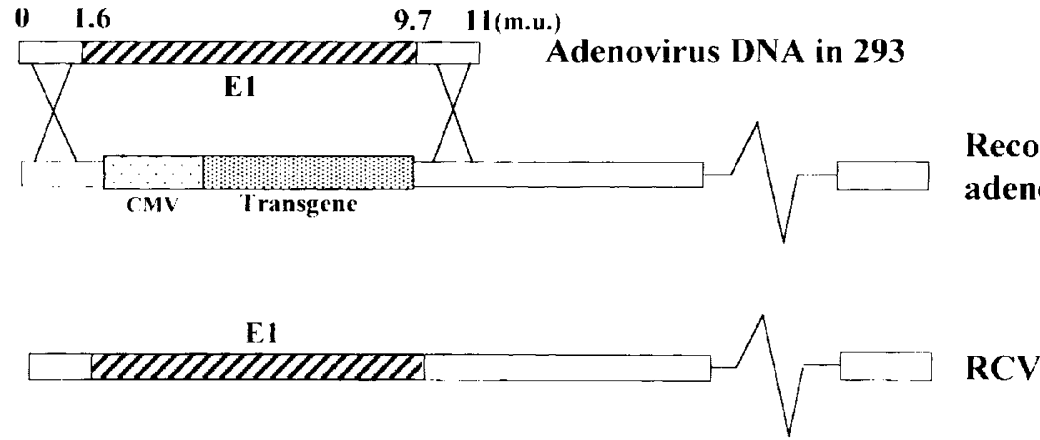

Figure 1. Scheme of RCV production mechanism by homologous recombination. Replication competent adenovirus is produced by homologous recombination between adenovirus gene of 293 and its homologous region of a recombinant adenovirus vector.

\section{Materials and Methods}

\section{Cell lines}

The cervical cancer cell line HeLa and the ovarian cancer cell line SKOV3 were maintained in Dulbecco's Modified Eagle's Medium (DMEM, Biowhittaker, Walkersville, MD, USA) supplemented with $10 \%$ fetal bovine serum. The human embryonic kidney cell line, 293 was maintained in Eagle's Minimum Essential Medium (EMEM, Biowhittaker, Walkersville, MD, USA) supplemented with $10 \%$ fetal bovine serum. All cell lines were obtained from ATCC (American Type Culture Collection, Rockville, MD, USA).

\section{Adenovirus- $\beta$ gal (Ad- $\beta$ gal) and Ad-p53}

The adenovirus containing wild-type p53 cDNA (Ad-p53) was constructed as described previously (Hwang et al., 1998). Briefly, the shuttle vector p $\delta \mathrm{A}-\mathrm{CMVp53}$ and an adenovirus vector pJM17 were used to cotransfect a packaging cell line, 293, by the calcium phosphate method. A replication deficient adenovirus clone was isolated and propagated in 293 cells. Ad-RSV- $\beta$ gal, a $\beta$ galactosidase-expressing adenovirus was kindly provided by Wei Wei Zhang (GenStar Therapeutics Corporation, San Diego, California, USA).

\section{Cloning of E1 and construction of HeLa-E1 cell line}

E1 gene of adenovirus was cloned from 293 genomic DNA by polymerase chain reaction (PCR) using a pair of E1 primers, 5'-CCGACACCGGGACTGAAAATGAG3' (542-564 bp) and 5'-CCACACATTTCAGTACCTCAATC-3' (3526-3504 bp). E1 PCR product was directly cloned into pCR3.1-Uni vector (Invitrogen, Groningen, Nether-lands) for eukaryotic gene expression. To construct a stable cell line, HeLa-E1, approximately $2 \times 10^{5}$ cells of HeLa were seeded in $60 \mathrm{~mm}$ dish before $24 \mathrm{~h}$ of transfection. Cells were transfected with FuGene6 reagent (Roche, Mannheim, Germany). After $48 \mathrm{~h}$ of transfection, transfected cells were screened with $400 \mu \mathrm{g} / \mathrm{ml}$ of geneticin (GIBCO-BRL, Gaithersburg,
MD, USA). Finally one clone was selected and propagated for further experiment.

\section{Western blot analysis}

Cells were lysed in RIPA buffer [50 mM Tris $(\mathrm{pH} 8.0)$, $150 \mathrm{mM} \mathrm{NaCl}, 1 \% \mathrm{NP} 40,0.1 \%$ SDS, and $0.5 \%$ sodiumdeoxycholate]. Equivalent amounts of whole cell extracts (10 $\mu \mathrm{g}$ for each; $5 \mu \mathrm{g}$ for 293) were resolved on $12 \%$ polyacrylamide gel and transferred to Hybond-ECL nitrocellulose filter membrane (Amersham, Buckinghamshire, UK). The membranes were blocked in $25 \mathrm{mM}$ Tris (pH 8.0) containing $125 \mathrm{mM} \mathrm{NaCl}, 0.1 \%$ Tween 20, and $5 \%$ skim milk. Protein bands were probed with either anti-E1A antibody (PharMingen, San Diego, CA. USA) or anti-p53 antibody (Novocastra, Newcastle, U.K.) followed by the labeling with horseradish per-oxidaseconjugated anti-mouse antibody (Amersham, Buckinghamshire, U.K). Bands were visualized using an ECL kit (Amersham, Buckinghamshire, U.K.) according to the manufacturer's instructions.

\section{Assay for replication competent virus (RCV)}

The generation of replication competent virus (RCV) in adenovirus produced either in HeLa-E1 or in 293 was detected by the presence of E1A gene in the DNA isolated from virus using PCR method. The sequences of $E 1 A$ primers and non-E1 primers were reported previously (Hwang et al., 1998). To check $\beta$-galactosidase gene, PCR was done using a pair of $\beta$-gal primers, 5 'CTGTATG-AACGGTCTGGTCT-3' (1803-1822 bp) and 5'-ATCTGTGAAAGAAAGCCTGA-3' (2303-2284 bp) generating 500 bp DNA segments containing $\beta$-galactosidase gene. Viral DNA was isolated by proteinase $\mathrm{K}(2$ $\mathrm{mg} / \mathrm{ml}$ ) treatment in the presence of $0.5 \%$ SDS followed by phenol extraction and ethanol precipitation.

\section{Results}

Establishment of HeLa cells which express E1 
HeLa-E1 was made by the introduction of E1 gene into cervical cancer cell line, HeLa. E1 gene of adenovirus type 5 was cloned by conventional PCR technique from 293 genomic DNA using primers described in Materials and Methods. The PCR product was directly cloned into pCR3.1-Uni vector for mammalian expression. After transfection into HeLa, cells were selected with geneticin $(400 \mu \mathrm{g} / \mathrm{ml})$. Finally one clone was isolated and propagated in $100 \mathrm{~mm}$ dish for further experiment. The expression of E1A gene in HeLa-E1 cells was confirmed by western blot analysis (Figure 2). HeLa-E1 cells appeared to induce an overexpression of E1A compared to the non-transfected HeLa cells as a control, which showed no E1A expression. However, the transient expression of E1A in HeLa induces a weak E1A expression.

\section{Replication deficient adenovirus production in HeLa-E1}

To demonstrate replication-deficient adenovirus production in HeLa-E1, we prepared adenovirus- $\beta$-galactosidase (Ad- $\beta$ gal), which is able to express $\beta$-galactosidase gene. To check RCV contamination of Ad- $\beta$ gal stock, viral DNA was isolated and PCR was done using non-E1 primers, $\beta$ gal primers and E1A primers (Figure

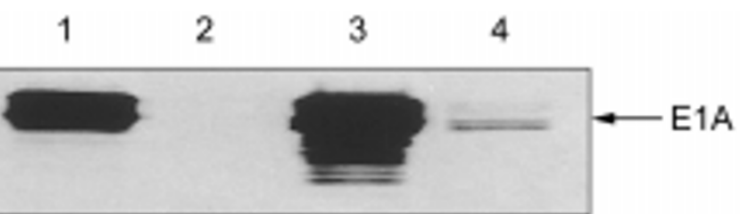

Figure 2. E1A expression in HeLa-E1 cell line. (Lane 1); 293 cell line, (Lane 2); HeLa cell line, (Lane 3); HeLa-E1 cell line, (Lane 4); HeLa cell line which is transiently transfected with E1. Each cell was collected and lysed in RIPA buffer. Equivalent amounts of whole cell extracts ( $10 \mu \mathrm{g}$ for each; $5 \mu \mathrm{g}$ for 293) were applied to western blot analysis for E1A detection.

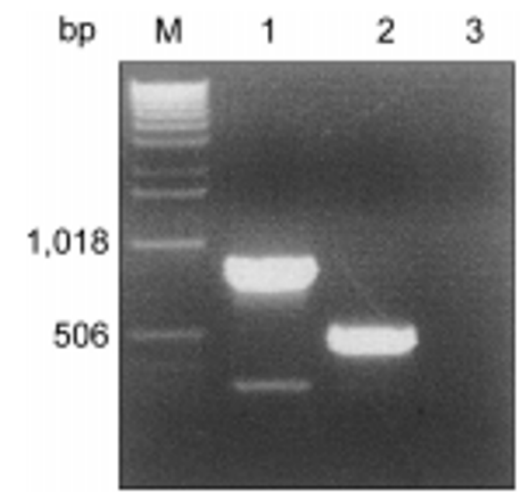

Figure 3. Identification of replication deficient adenovirus by PCR. PCR was done using the DNA extracted from the Ad- $\beta$ gal with non-E1 adenovirus primers (lane 1), $\beta$ gal primers (lane 2) and E1A primers (lane 3). A 861 bp of non-E1 region and $500 \mathrm{bp}$ of $\beta$ gal region were amplified whereas a 752 bp of $\mathrm{E} 1 \mathrm{~A}$ was not produced.
3). E1A fragment was not detected, instead DNA bands for non-E1 and $\beta$-galactosidase gene was generated as $861 \mathrm{bp}$ and $500 \mathrm{bp}$ fragments, respectively. These results indicate that the clone selected does not contain any RCV. Replication deficient Ad- $\beta$ gal was infected into HeLa-E1 or 293 cells with 100 m.o.i (multiplicity of infection). After $48 \mathrm{~h}$ of infection, culture media and pellet were collected and separated by centrifugation. Adenovirus DNA was extracted from the supernatant, and then PCR was done to detect RCV production with primers described in Materials and Methods. No RCV production was detected in Ad- $\beta$ gal produced in HeLa-E1, whereas Ad- $\beta$ gal produced in 293 cells contained RCV (Figure 4A). To confirm the generation of RCV during successive adenoviral production, the rest of supernatant was used for second infection. After $48 \mathrm{~h}$ of second infection, adenovirus DNA was isolated from the supernatant. When PCR was done with same primers as described above, again there was no RCV production in Ad- $\beta$ gal produced in HeLa-E1 in contrast to in 293 (Figure 4B). These results clearly demonstrate that the production of adenovirus vector using HeLa-E1 cells excludes the generation of RCV.

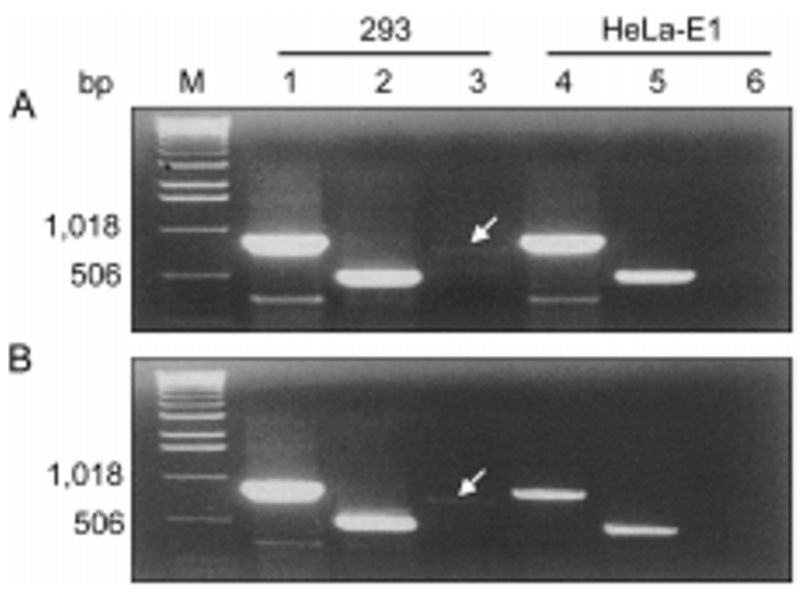

Figure 4. Test for the presence of replication competent virus in Ad- $\beta$ gal produced in HeLa-E1 or 293. PCR was done using the viral DNA of Ad- $\beta$ gal produced in 293 or HeLa-E1 after first infection (panal A) and second infection (panal B) with non-E1 primers (lane 1, 4), Bgal primers (lane 2, 5) and E1A primers (lane 3,6 ). E1A fragments produced in 293 are indicated by arrows.

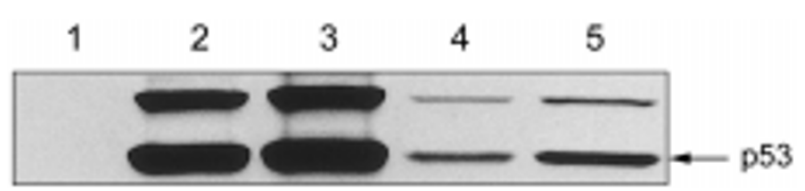

Figure 5. p53 expression by Ad-p53 infection in SKOV3 cell line. SKOV3 was treated with PBS (lane1) or supernatant of Ad-p53 produced in 293 (Lane 2, 3) or HeLa-E1 (lane 4,5). 1.5ml supernatant(lane 2, 4) or $3 \mathrm{ml}$ supernatant (Lane 3,5$)$ of Ad-p53 was used for each infection. Each sample was collected and lysed in RIPA buffer. Each of 10 ug protein was applied for western blot analysis for p53 detection. 


\section{Identification of adenovirus-p53 produced in HeLa- E1}

To demonstrate the expression of p53 gene, an ovarian cancer cell line, SKOV3 was infected with adenovirusp53 produced in either HeLa-E1 or 293 cells. After $48 \mathrm{~h}$ of infection, total cells were lysed for whole cell extracts and then western blot analysis was done with anti-p53 antibody. As shown in Figure 5, a high level expression of p53 protein was induced in SKOV3 cells transfected with Ad-p53 that was generated in HeLa-E1 or 293. However, there was no detectable expression of p53 protein in SKOV3 treated with PBS as a negative control. Taken together, these results indicate that HeLa-E1 can be used for packaging cell line for the generation of replication-deficient recombinant adenovirus. It is noteworthy that HeLa-E1 shows lower productivity than 293 as a packaging cell line. However, this problem can be overcome by selecting stable cell lines which contain many E1 gene copies in their genome. The most critical point is that this strategy can be used for making packaging cell lines that exclude RCV generation during recombinant adenovirus propagation.

\section{Discussion}

The most widely used adenovirus packaging cell line is 293, which was derived from human embryonic kidney (HEK) cells by transformation with Ad5 fragments. 293 cells contain the left $11 \%$ of Ad5 genome. RCV is produced by homologous recombination between Ad5 fragment in 293 cells and recombinant adenovirus vector during multiple passages. To solve this RCV problem, we proposed a new strategy to make a packaging cell line for RCV-free adenovirus propagation. We selected a cervical cancer cell line HeLa to develop a new packaging cell line for RCV-free recombinant adenovirus production because HeLa shows high susceptibility to adenovirus relative to other stable cell lines. HeLa-E1 was made by the introduction of minimum E1 (1.6-9.7 m.u.) into HeLa. When HeLa-E1 cells were employed as packaging cells, there was no detectable RCV generation during recombinant adenovirus production, as determined by the PCR analysis. On the contrary, an easily detectable level of RCV appeared to be generated during the first propagation in 293 cells. Although several previous reports showed that RCV was produced at the late passage during large-scale or successive production of recombinant adenovirus in 293 cells (Lochmuller et al., 1994), in our experiment, RCV generation was detected from the early passage. This is due to the possibility that RCV can be produced in any passage duirng the propagation in 293 cells. In addition, we confirmed that a recombinant adenovirus possessing p53 gene (Ad-p53) produced in HeLa-E1 cloud be successfully transfected into a human ovarian cancer cell line SKOV3, and induce an overexpression of p53 protein, suggesting that a new packaging cell line HeLa$\mathrm{E} 1$ is applicable as a packaging cell line for the production of RCV-free recombinant adenovirus. However, since the produc-tivity of recombinant adenovirus in HeLa-E1 is lower than in 293 cells, it needs to be further studied to increase the productivity. It is highly likely that a possible approach to overcome this problem may be simply to select a cell line that contains many E1 gene copies in their genome like 293 cell line.

In conclusion, in this report, we proposed a strategy for preparing a packaging cell line which can exclude RCV generation and thus its contamination during largescale as well as multiple-passage production of recombinant adenovirus. This strategy could be applicable to normal cells because the expression of E1 gene by itself is able to transform normal cells to immortalized cells as 293 cell lines. However, we need to handle many obstacles to apply this strategy to the gene therapy for human. Particularly, at first we should make a packaging cell line derived from normal human cells, because we cannot use any products obtained from cancer cells for the human gene therapy. In addition, we should consider the virus productivity, life cycle, and longevity of cells when we prepare a packaging cell line.

\section{Acknowledgement}

We thank Dr. Weonmee Park and Dr. Eun-Sung Hwang for reviewing this manuscript and for helpful discussion. This work was supported by a G7 grant from the Korean Ministry of Science and Technology (98-G-08-02-A-11) to J.H.L. and Samsung grant \#SBRI C-99-001-1 to J.H.L.

\section{References}

Amalfitano A, Begy CR, Chamberlain, JS. Improved adenovirus packaging cell lines to support the growth of replicationdefective gene-delivery vectors. Proc Natl Acad Sci USA 1996;93:3352-56

Berkner KL, Sharp PA. Generation of adenovirus by transfection of plasmids. Nucleic Acids Res 1983;17:6003-21

Berkner KL. Development of adenovirus vectors for the expression of heterologous genes. Biotechniques 1988;6:616-29

Bett AJ, Krougliak V, Graham FL. DNA sequence of the deletion/insertion in early region 3 of Ad5 dl309. Virus Research 1995;39:75-82

Caravokyri C, Leppard KL. Constitutive episomal expression of polypeptide IX(pIX) in a 293-based cell line complements the deficiency of pIX mutant adenovirus type5. J Virol 1995; 69:6627-33 
Chinnadurai, G., Chinnadurai, S, Brussa, J. Physical mapping of a large-plaque mutation of adenovirus type 2. J Virol 1979;32:623-28

Choi JH, Ahn KS, Kim J, Hong YS. Enhanced induction of Bax gene expression in $\mathrm{H} 460$ and $\mathrm{H} 1299$ cells with the combined treatment of cisplatin and adenovirus mediated wtp53 gene transfer. Exp Mol Med 2000;32:23-28

Fallaux FJ, Kranenburg O, Cramer SJ, Houweling A, Van Ormondt, H, Hoeben, RC. Characterization of 911:A new helper cell line for the titration and propagation of early region 1-deleted adenoviral vectors. Human Gene Ther. 1996;7:21522

Forsayeth JR, Garcia PD. Adenovirus-mediated transfection of cultured cells. Biotechniques 1994;17:354-56

Gao G-P, Engdahl RK, Wilson JM. A cell line for high-yield production of E1-deleted adenovirus vectors without the emergence of replication-competent virus. Hum Gene Ther 2000; 11:213-19

Graham FL, Smiley J, Russel WC, Nairn R. Characteristics of a human cell line transformed by DNA from human adenovirus type 5. J Gen Viol 1977;36:59-74

Graham FL, Prevec L. Methods for construction of adenovirus vectors. Mol Biotechnol 1995;3:207-20

He TC, Zhou S, Da Costa LT, Yu J, Kinzler KW, Vogelstein, B. A simplified system for generating recombinanat adenoviruses. Proc Natl Acad Sci USA 1998;95:2509-14

Horwitz MS. Adenoviridae and their replication. In virology. Fields BN, Knipe DM, Chanoch RM, Hirsch MS, Melnick YL, Monath TP, Roizman B (Eds.), pp.1679-1721, Raven Press Ltd, New York, 1990.

Hwang ES, Kim J, Kim JS, Kao C, Ko S-C, Chung L, Lee J$\mathrm{H}$. The effects of the adenoviral-mediated wild-type p53 delivery in human epithelial ovarian cancer cell lines in vitro and in vivo. Int J Gynecol Cancer 1998;8:27-36
Kim J, Hwang ES, Kim JS, You EH, Lee SH, Lee JH. Intraperitoneal gene therapy with adenoviral-mediated p53 tumor suppressor gene for ovarian cancer model in nude mouse. Cancer Gene Ther 1999;6:172-78

Lochmuller $H$, Jani $A$, Huard J, Prescott $S$, Simoneau M, Massie B, Karpati G, Acsadi G. Emergence of early region 1containing replication-competent adenovirus in stocks of replication-defective adnovirus recombinants (dE1+dE3) during multiple passages in 293 cells. Hum Gene Ther 1994;5:148591

Shenk T, Jones N, Colby W, Fowlkers D. Functional analysis of adenovirus-5 host-range deletion mutants defective for transformation of rat embryo cells. Cold Spring Harbor Symp Quant Biol 1979;44:367-75

Smith TA, Mehaffey MG, Kayda DB, Saunders JM, Yei S, Trapnell, BC, McClelland, A, Kaleko, M. Adenovirus-mediated expression of therapeutic plasma levels of human factor IX in mice. Nature Genet 1993;5:397-402

Wang $\mathrm{Q}$, Jia X-C, Finer $\mathrm{MH}$. A packaging cell line for propagation of recombinant adenovirus vectors containing two lethal gene-region deletions. Gene Ther 1995;2:775-83

Yang Y, Nunes FA, Berencsi K, Furth EE, Gonczol E, Wilson JM. Cellular immunity to viral antigens limits E1-deleted adenoviruses for gene therapy. Proc Natl Acad Sci USA 1994;91:4407-11

Zhang WW, Fang X, Branch CD, Mazur W, French BA, Roth JA. Generation and identification of recombinant adenovirus by liposome-mediated transfection and PCR analysis. Biotechniques 1993;15:868-71

Zhang WW, Koch PE, Roth JA. Detection of wild-type contamination in a recombinant adenoviral preparation by PCR. Biotechniques 1995;18:444-47

Zhang WW. Development and application of adenoviral vectors for gene therapy of cancer. Cancer Gene Ther 1999;6:113-38 